\title{
De novo COX2 mutation in a LHON family of Caucasian origin: implication for the role of mtDNA polymorphism in human pathology
}

Received: 8 September 2005/ Accepted: 31 October 2005/Published online: 18 January 2006

(C) The Japan Society of Human Genetics and Springer-Verlag 2006

\begin{abstract}
Recent studies suggest that certain mutations with phylogeographic importance as haplogroup markers may also influence the phenotypic expression of particular mitochondrial disorders. One such disorder, Leber's hereditary optic neuropathy (LHON), demonstrates a clear expression bias in mtDNAs belonging to haplogroup J, a West Eurasian maternal lineage defined by polymorphic markers that have been called 'secondary' disease mutations. In this report, we present evidence for a de novo heteroplasmic COX2 mutation associated with a LHON clinical phenotype. This particular mutation - at nucleotide position 7,598 - occurs in West Eurasian haplogroup $\mathrm{H}$, the most common maternal lineage among individuals of European descent, whereas previous studies have detected this mutation only in East Eurasian haplogroup E. A review of the available mtDNA sequence data indicates that the COX2 7598 mutation occurs as a homoplasic event at the tips of these phylogenetic branches, suggesting that it could be a variant that is rapidly eliminated by selection. This finding points to the potential background influence of polymorphisms on the expression of mild deleterious mutations such as LHON mtDNA defects and
\end{abstract}

\author{
S.I. Zhadanov $(\bowtie) \cdot$ T.G. Schurr \\ Department of Anthropology, University of Pennsylvania, \\ 325 University Museum, 3260 South Street, Philadelphia, \\ PA 19104-6398, USA \\ E-mail: zhadanov@sas.upenn.edu \\ Tel.: + 1-215-5732656 \\ Fax: +1-215-8987462 \\ S.I. Zhadanov \\ Institute of Cytology and Genetics, \\ SB Russian Academy of Sciences, \\ Novosibirsk, Russia \\ V.V. Atamanov \\ Sv. Fyodorov State Institution, IRTC Eye Microsurgery, \\ Novosibirsk, Russia \\ N.I. Zhadanov \\ Dovolnoe District Hospital, Novosibirsk Region, \\ Dovolnoe, Russia
}

further highlights the difficulties in distinguishing deleterious mtDNA changes from neutral polymorphisms and their significance in the development of mitochondriopathies.

Keywords Leber's hereditary optic neuropathy · COX2 - Haplogroup marker - mtDNA polymorphism · Mitochondriopathy

\section{Introduction}

Leber's hereditary optic neuropathy [MIM 535000] (LHON), one of the most frequent mitochondrial disorders (Man et al. 2003), is a common cause of blindness in young males. While three mtDNA missense mutations at nucleotide positions 3460A/ND1, 11778A/ND4 and $14484 \mathrm{C} / \mathrm{ND} 6$ are traditionally considered to be strong risk factors for visual loss, LHON represents an example of a mitochondriopathy that demonstrates significant variability in its expression in different populations, as well as individual genetic backgrounds. Known features of LHON, including incomplete penetrance, genderspecific bias, often inconsistent biochemical profiles, and large numbers of cases without any common pathogenic mtDNA mutation, all suggest a considerable heterogeneity in disease expression and etiology, and may reflect the involvement of other genetic and environmental factors in disease development.

Numerous studies have demonstrated that cases of LHON are also associated with a particular West Eurasian mtDNA background-haplogroup $\mathrm{J}$-more frequently than expected based on normal control haplogroup frequencies and a random pattern of pathogenic mutation distribution in Caucasians. Some of the phylogenetically ancient polymorphic sites in haplogroup $\mathbf{J}$ have been suggested to play a secondary role in LHON expression (see, e.g., Brown et al. 1995; Torroni et al. 1997). Investigation of this interaction between LHON mutations and different genetic environments 
has provided new insights into disease-causing loci, and revealed the existence of other, hitherto unknown, mechanisms for the pathogenesis of mitochondriopathies (Valentino et al. 2004; Zhadanov et al. 2005).

A mitochondrial enzyme complex that has received considerable attention in studies seeking a greater understanding of mitochondrial pathogenesis is cytochrome $c$ oxidase (COX). $\mathrm{COX}$ is the terminal electron acceptor of the mitochondrial respiratory chain that transfers electrons from reduced cytochrome $c$ to oxygen to produce water, coupling this exchange with the pumping of protons from the mitochondrial matrix towards the intermembrane space (Babcock and Wikstrom 1992). COX deficiencies have been described as a cause of a wide spectrum of mitochondriopathies, and may be associated with mutations in both mitochondrial and nuclear DNAs (for a review, see Darin et al. 2003). In a few early studies, homoplasmic mutations in the mtDNA-encoded subunit were reported as the possible cause of LHON, but their role is still disputed (Johns and Neufeld 1993; Johns et al. 1994).

In this report, we describe a heteroplasmic 7598A mutation in the fifth codon of the COX2 subunit associated with LHON in an affected family of Caucasian origin. Genetic screening of maternal relatives for this variant proved its de novo occurrence in the pedigree of the studied family. The 7598A mutation has been previously described as a haplogroup-specific marker for East Eurasian haplogroup E, a monophyletic sub-branch of mtDNA haplogroup M9 that appears at minor frequencies in some East Asian populations (Stoneking et al. 1990; Ballinger et al. 1992; Ingman and Gyllensten 2003). However, it also occur as homoplasmic events in two phylogenetic branches within Caucasian populations (Finnila et al. 2001; Howell et al. 2003). Interestingly, this mutation has arisen twice in haplogroup $\mathrm{H}$ mtDNAs, including the case that is the focus of our study, and, in both instances, was associated with LHON expression, implying that it might have some role in disease etiology.

This finding is consistent with the hypothesis that, once occurring in a specific genetic background, certain polymorphic mutations can contribute to mechanisms influencing development of mitochondriopathies. It provides additional suggestive evidence for functional differences among mtDNA haplogroups, and the possible rapid elimination of deleterious genetic variants by selection. We discuss the possible pathogenic role of the 7598A mutation, as well as describe the complexity of population genetic factors that might influence LHON expression.

\section{Materials and methods}

Patients

All investigations were conducted with the approval of the ethics committees of the participating institutions and according to the tenets of the Declaration of Helsinki. Informed consent was obtained from all individuals prior to donation of blood samples and genetic analysis of their mtDNAs. The proband and his mother were interviewed about their history of symptoms and genealogy, and were also given standard neurological and ophthalmological exams by experienced clinicians. Best-corrected visual acuity (BCVA) was measured with the Snellen chart, together with far and near binocular visual acuity. Colour vision was assessed with the Farnsworth desaturated D15 or Ishihara tests. Visual fields were tested with Goldmann and Humphrey perimetry, and slit-lamp microscopic examinations performed before and after dilation of the pupils. Brain magnetic resonance imaging (MRI), electroencephalography (EEG), electrocardiography (EKG) and follow-up blood biochemistry were also carried out using standard methods. The proband and his mother declined further invasive investigation, thus, muscle biopsies were not performed.

\section{Molecular genetic studies}

Venous blood samples were obtained from the proband and his mother, and hair samples from family member IV-16 (see Fig. 1) were also acquired. Total genomic DNA was extracted from the samples by a standard phenol-chloroform technique. Initially, patient $\mathrm{mtD}$ NAs were screened for a comprehensive list of reported deleterious mtDNA changes, including three common LHON mutations (3460A, 11778A and 14484C), by PCR amplification followed by mutation-specific restriction endonuclease digestion (Brown et al. 1995). Southern blotting of mtDNAs subjected to SnaBI and $P v u$ II enzyme digestion was performed to rule out the presence of common mtDNA deletion/rearrangements.

The coding region of the proband's mtDNA was subsequently amplified in 3-4 kb overlapping fragments with specific primer sets (primer sequences available upon request). Both strands were sequenced using BigDye Terminator Pre-Mix kits, v3.1 (Applied Biosystems, Foster City, CA), purified with Centri-Sep columns (Princeton Separations, Adelphia, NJ), and run on ABI 3100 Gene Analyzers in the Sequencing Centre of the Department of Genetics, University of Pennsylvania. Trace files were aligned with Sequencher 3.1 (GeneCodes, Ann Arbor, MI) and compared to Cambridge Reference Sequence (rCRS) (Anderson et al. 1981; Andrews et al. 1999). The presence of the 7598A mutation was verified using a mutation-specific restriction digestion with HhaI following PCR amplification of mtDNA with a specific primer set (see Fig. 3b). In addition, we screened 187 haplogroup-matched healthy and mitochondrial disease controls available from our ongoing biomedical projects for this COX2 mutation.

\section{Quantification of DNA levels}

The amount of mutated mtDNA present in blood and hair samples was quantified using restriction enzyme 


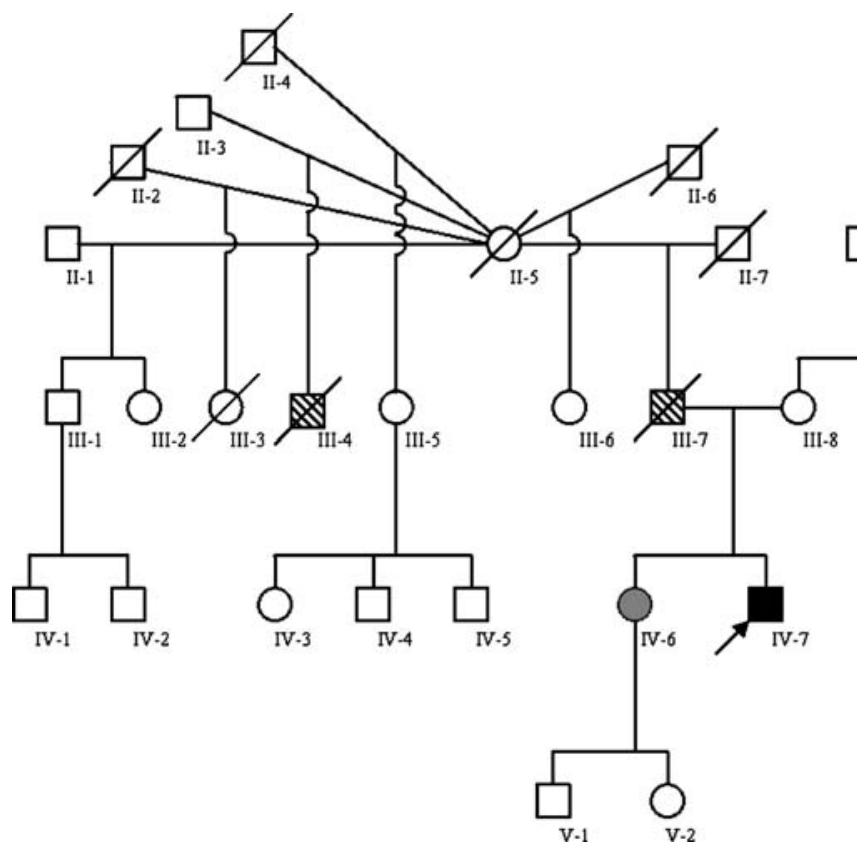

Fig. 1 The family pedigree of the Leber's hereditary optic neuropathy (LHON) proband. The proband is indicated by the arrow. Patients affected with only visual and neurological symp-

analysis. Digests were electrophoresed in agarose gels and analysed by laser densitometry after ethidium bromide staining. The level of heteroplasmy was estimated from actual pixel intensity of different bands on the gel image (Liu et al. 1997).

Sequence alignment and molecular modelling

To exclude potential nuclear pseudogene amplification, a primer set was designed to avoid significant homology with the human nuclear genome. Direct BLAST search (Altschul et al. 1997) of the National Centre for Biotechnology Information sequence database for the human genome using primer sequences as queries did not produce any structurally reasonable hits other than in mitochondrial DNA, regardless of the search parameters (http://www.ncbi.nlm.nih.gov/BLAST/).

\section{Protein secondary structure}

The protein secondary structure of COX2 was analysed using computer-assisted Proteomics tools of the ExPASy server and mtSNP Database. A total of 974 amino acid sequences were retrieved from GenBank and aligned using CLUSTAL $\mathrm{W}$, as provided in the Biology Workbench (http://workbench.sdsc.edu). The selected sequences aligned included those from Homo sapiens, Hylobates lar, Bos taurus, Equus caballus, Ovis aries, Sus scrofa, Pteropus scapulatus, Echinops telfairi, Rattus norvegicus, Mus musculus, Ochonota collaris, Gallus gallus, sea urchin and Drosophila.

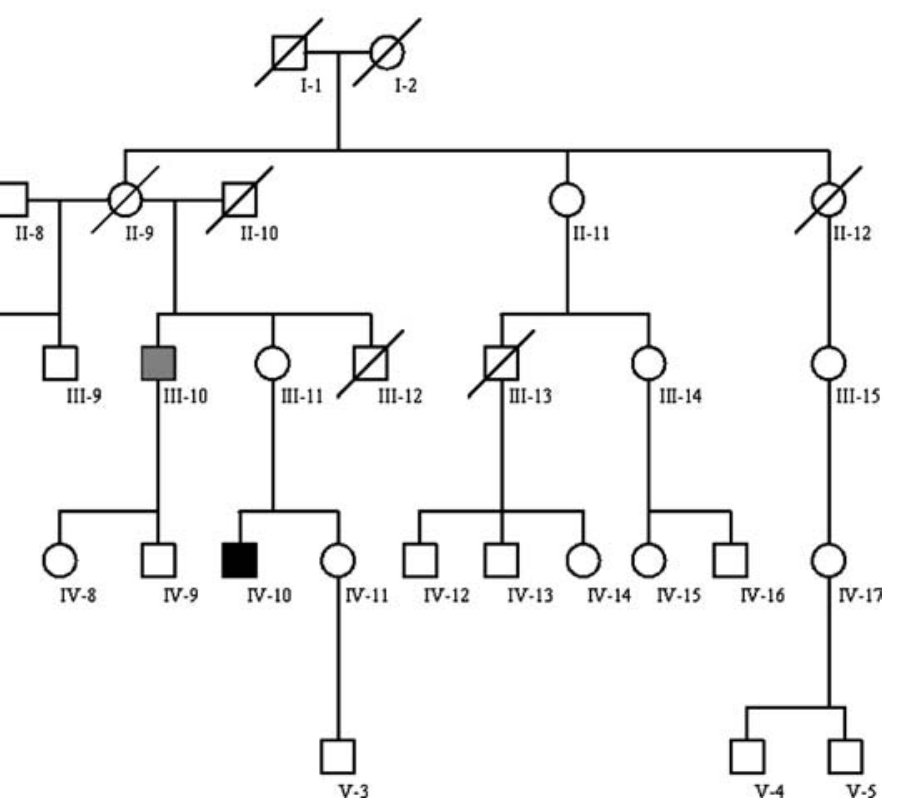

toms are indicated by black and grey symbols, respectively. Individuals suffering from hypertrophic cardiomyopathy and congestive heart failure are shown by hatched symbols

Phylogenetic analysis

The mtDNA data from the proband's family and control population studies were subjected to phylogenetic analysis with NETWORK (Bandelt et al. 1995) to determine the evolutionary relationships among haplotypes bearing the 7598A mutation. The haplotype network was initially constructed with the reduced-median algorithm (Bandelt et al. 1995) and then re-drawn by hand.

\section{Results}

\section{Clinical features}

An otherwise healthy 26-year-old Caucasian male patient (Fig. 1, IV-7) experienced painless blurring of vision in his left eye at age 24 , with the subsequent involvement of the second eye within a 6-month period. Initial examination records provided evidence for hyperemia, swelling of papillae, and diffusion of disk verges that were recognised as acute optic neuropathy. His vision progressively deteriorated until, a year after initial onset, his BCVA stabilised at 0.01 binocularly, with extended central scotomas in both visual fields. During the following 2 years, he was given a few courses of non-invasive low frequency optic nerve electrostimulation, and, by the time he was referred for our investigation, his BCVA had improved to 0.02 in his left eye and 0.2 in his right eye.

Ophthalmoscopic findings showed him to have isolated temporal pallor of optic discs bilaterally, while the 
peripheral retina and fundus $a / v$ ratio were unremarkable. On visual field examination, central scotomas were found to be reduced in size relative to earlier records, with a lower number of absolute defects on computerised Humphrey Field Analyser. Colour vision testing revealed non-specific trichromatic reduction involving all primary axes. The flash ERG appeared normal, reflecting unaffected functional activity of photoreceptors and pigment epithelium.

At age 22, 2 years prior to the worsening of his vision, the patient experienced two consecutive episodes of generalised seizures, and was diagnosed as having grandmal epilepsy. He was effectively treated with carbamazepine without recurring symptoms over the subsequent years. Upon neurological examination, he showed no evidence of muscle weakness or fatigue, and all of his tendon reflexes were normal. The results of brain MRI and EEG were unremarkable, as were basic biochemical tests. Electrocardiography revealed him to have sinus bradycardia of $50 \mathrm{bpm}$.

His family history was notable for having individuals expressing a variety of optic and neurological symptoms. Clinical examination of the proband's mother (III-8) revealed no pathological eye or neurological signs, aside from mild hyperopia with BCVA of 0.9/1.0. However, the proband's first cousin (IV-10) experienced severe visual deterioration at age 20 as a result of bilateral optic neuropathy. Despite intense anti-inflammatory therapy, his visual acuity remained non-correctable at low levels. In addition, an uncle on the proband's mother's side (III10) was described as suffering from repeated migraine episodes, focal seizures and pyramidal syndrome since his youth. While otherwise appearing healthy, the proband's blood-sister (IV-6) was also reported as having experienced severe migraine headaches.

On the proband's father's side, a few family members were diagnosed with X-linked inherited hypertrophic cardiomyopathy. In addition, both the proband's father (III-7) and his half-blood brother (III-4) died from congestive heart failure in their middle thirties. It is notable that the proband's paternal grandmother (II-5) also developed heart muscle hypertrophy at her late fifties. However, she also had a history of hypertension, and died from brain infarction at the age of 60 . All other female individuals in this branch of the family pedigree appeared to be healthy, although they were possibly carriers of the X-linked defect.

\section{Genetic and phylogenetic analysis}

Southern blot analysis excluded the presence of largescale rearrangements of mtDNA in total genomic DNA from the patient's blood (data not shown). Mutationspecific RFLP-PCR analysis of the proband's mtDNA did not reveal any common or rare LHON mutations. The coding sequence of the proband's mitochondrial genome revealed eight base changes compared to the rCRS (Anderson et al. 1981) (Table 1). This mtDNA belonged to West Eurasian haplogroup $\mathrm{H}$ due to the presence of lineage-specific polymorphisms at nucleotide positions 2706A, 7028C, 11719G and 14766C, and lacked any other European haplogroup-specific polymorphisms (Torroni et al. 1996; Herrnstadt et al. 2002).

The proband's mtDNA also carried a few rare genetic variants. Of these, the synonymous $3333 \mathrm{~T}$ transition in the ND1 gene apparently represented a silent mutation. It was detected in two of our haplogroup $\mathrm{H}$ controls, and had been noted in four other haplogroup $\mathrm{H}$-specific mtDNA sequences in extensive European population surveys, two of which also had the 11778A LHON mutation in their mtDNAs (Herrnstadt et al. 2002; Howell et al. 2003) (Fig. 2). Thus, the 3333T mutation likely represents an ancestral variant, and delineates a separate monophyletic subcluster within this haplogroup.

Among mtDNAs belonging to haplogroup $\mathrm{H}$, the 3849A transition was unique to the proband's haplotype (Fig. 2), although it had previously been reported in healthy controls whose mtDNAs belonged to haplogroups U2 and R (Herrnstadt et al. 2002; Pala-
Table 1 Mitochondrial DNA (mtDNA) nucleotide changes in the coding region of the studied case family compared to the revised Cambridge sequence (adapt from Anderson et al. 1981; Andrews et al. 1999). Polymorphic sites were determined by searching the MITOMAP database (http://www.mitomap.org), unless otherwise specified

\begin{tabular}{rllll}
\hline Nucleotide position & Nucleotide change & Region/gene & Amino acid change & Comment \\
\hline 750 & A to G & 12S rRNA & - $^{\text {a }}$ & CRS polymorphism \\
1,438 & A to G & 12S rRNA & - & CRS polymorphism \\
3,333 & C to T & ND1 & - & Polymorphism \\
3,849 & G to A & ND1 & Current study \\
4,769 & A to G & ND2 & Ala to Thr & CRS polymorphism \\
7,598 & G to A & COX2 & Current study \\
8,860 & A to G & ATP6 & Thr to Ala & CRS polymorphism \\
15,326 & T to C & CytB & Thr to Ala & CRS polymorphism \\
\hline
\end{tabular}

\footnotetext{
${ }^{\mathrm{a}}$ No amino acid change

${ }^{b}$ Found in downloaded mtDNA sequences (adapt from Herrnstadt et al. 2002; Howell et al. 2003), and present in haplogroup H specific background in our sample set

${ }^{\mathrm{c}}$ Heteroplasmic appearance; also reported as homoplasies in different phylogenetic branches (see text for details); CRS polymorphism refers to rare polymorphisms present in the original Cambridge sequence (adapt from Anderson et al. 1981; Andrews et al. 1999)
} 
Fig. 2 A diagram showing the phylogenetic relationships between different haplotypes carrying the 7598A mutation. Coding region sequence data only were used to construct these branches. $\mathrm{LHON}^{1}-$ $\mathrm{LHON}^{4}$ represents LHON patient haplotypes. LHON ${ }^{1}$, LHON $^{2}$ and LHON ${ }^{4}$

correspond to S003, S136 and S015, respectively (from Howell et al. 2003). $\mathrm{LHON}^{3}$ is the proband from this study. The asterisk $(*)$ indicates the heteroplasmic appearance of the 7598A mutation. $\mathrm{C} 1-7$ are control samples. $\mathrm{C} 1$ and $\mathrm{C} 3$ were taken from our sample set; $\mathrm{C} 2, \mathrm{C} 4$ and $\mathrm{C} 6$ are haplotypes $\# 74,116$, and 214 from Herrnstadt et al. (2002); and the C5 haplotype is sample \#153 from Finnila et al. (2001). C7 corresponds to GenBank accession number AY289070 (adapted from Ingman and Gyllensten 2003). The West Eurasian portion of the phylogeny is enclosed in the dashed square

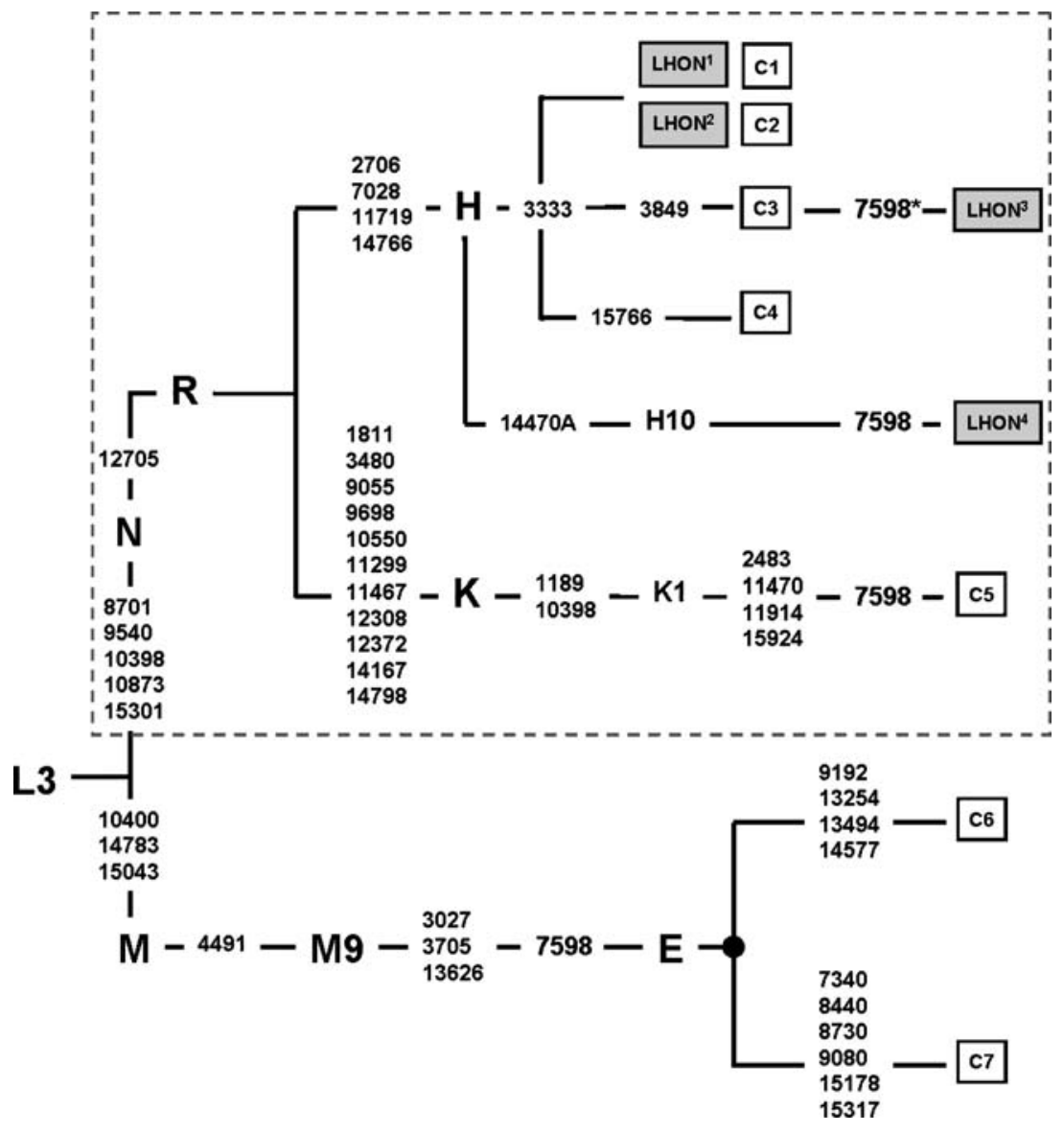

nichamy et al. 2004). This mutation modified the third nucleotide of a leucine codon in the ND1 gene and did not create an amino acid substitution, and thus is a silent base change.

The only candidate mutation - a G $\rightarrow$ A transition at 7,598 - altered the very beginning of the COX2 gene sequence (Table 1). At the translational level, this transition substitutes a small hydrophobic neutral alanine

Table 2 Evolutionary conservation of the A5T residue in 7598Apositive Leber's hereditary optic neuropathy (LHON) family and a number of other species. The altered amino acid has only low evolutionary conservation but a hydrophobic residue (indicated in bold) is present at this position in most of these species, and is for a hydrophilic polar threonine at the fifth amino acid position of the COX2 subunit, thereby changing its sidechain hydrophobicity. Phylogenetic analysis of COX2 polypeptide sequences from different species demonstrated that the alanine residue is relatively poorly conserved, although it is adjacent to a highly conserved, and hydrophilic, glutamine residue in an evolutionarily stable domain (Table 2). Interestingly, the mtDNA of the

adjacent to a highly conservative glutamine. Data were taken from ExPASy Proteomics (http://us.expasy.org/cgi-bin/protscale.pl) and mtSNP database (http://www.giib.or.jp/cgi-bin/mtsnp/specAligh/ ctrlSpecAlignE.cgi)

\begin{tabular}{|c|c|c|c|c|c|c|c|c|c|c|c|}
\hline LHON family & M & A & $\mathrm{H}$ & A & $\mathrm{T}^{\mathrm{a}}$ & $\mathrm{Q}$ & $\mathrm{V}$ & G & $\mathrm{L}$ & Q & $\mathrm{D}$ \\
\hline Homo sapiens & M & A & $\mathrm{H}$ & A & A & $\mathrm{Q}$ & $\mathrm{V}$ & $\mathrm{G}$ & $\mathrm{L}$ & $\mathrm{Q}$ & $\mathrm{D}$ \\
\hline Hylobates lar & M & A & $\mathrm{H}$ & A & $\mathrm{T}$ & $\mathrm{Q}$ & V & G & $\mathrm{L}$ & $\mathrm{Q}$ & $\mathrm{D}$ \\
\hline Bos taurus & M & A & $\mathrm{Y}$ & $\mathrm{P}$ & M & $\mathrm{Q}$ & $\mathrm{V}$ & G & $\mathrm{L}$ & $\mathrm{Q}$ & $\mathrm{D}$ \\
\hline Ovis aries & $\mathrm{M}$ & A & $\mathrm{Y}$ & $\mathrm{P}$ & M & $\mathrm{Q}$ & $\mathrm{L}$ & G & $\mathrm{F}$ & Q & $\mathrm{D}$ \\
\hline Equus caballus & M & A & $\mathrm{Y}$ & $\mathrm{P}$ & $\mathrm{F}$ & $\mathrm{Q}$ & $\mathrm{L}$ & G & $\mathrm{F}$ & $\mathrm{Q}$ & $\mathrm{D}$ \\
\hline Sus scrofa & $\mathrm{M}$ & A & $\mathrm{Y}$ & $\mathrm{P}$ & $\mathrm{F}$ & $\mathrm{Q}$ & $\mathrm{L}$ & G & $\mathrm{F}$ & $\mathrm{Q}$ & $\mathrm{D}$ \\
\hline Pteropus scapulatus & M & A & $\mathrm{Y}$ & $\mathrm{P}$ & $\mathrm{S}$ & $\mathrm{Q}$ & $\mathrm{L}$ & G & $\mathrm{F}$ & $\mathrm{Q}$ & $\mathrm{D}$ \\
\hline Echinops telfairi & M & A & $\mathrm{Y}$ & $\mathrm{P}$ & $\mathrm{S}$ & $\mathrm{Q}$ & $\mathrm{L}$ & G & $\mathrm{F}$ & $\mathrm{Q}$ & $\mathrm{D}$ \\
\hline Rattus norvegicus & $\mathrm{M}$ & A & $\mathrm{Y}$ & $\mathrm{P}$ & $\mathrm{F}$ & $\mathrm{Q}$ & $\mathrm{L}$ & G & $\mathrm{L}$ & Q & $\mathrm{D}$ \\
\hline Mus musculus & M & A & Y & $\mathrm{P}$ & $\mathrm{F}$ & $\mathrm{Q}$ & $\mathrm{L}$ & G & $\mathrm{L}$ & Q & $\mathrm{D}$ \\
\hline Ochotona collaris & M & A & $\mathrm{Y}$ & $\mathrm{P}$ & $\mathrm{S}$ & $\mathrm{Q}$ & $\mathrm{M}$ & G & $\mathrm{F}$ & Q & $\mathrm{D}$ \\
\hline Gallus gallus & $\mathrm{M}$ & A & $\mathrm{N}$ & $\mathrm{H}$ & $\mathrm{S}$ & $\mathrm{Q}$ & $\mathrm{L}$ & G & $\mathrm{F}$ & Q & $\mathrm{D}$ \\
\hline Sea urchin & M & G & $\mathrm{T}$ & W & A & $\mathrm{Q}$ & $\mathrm{F}$ & G & $\mathrm{L}$ & $\mathrm{Q}$ & $\mathrm{D}$ \\
\hline Fruit fly & M & $\mathrm{S}$ & $\mathrm{T}$ & W & A & $\mathrm{N}$ & $\mathrm{L}$ & G & $\mathrm{L}$ & Q & $\mathrm{D}$ \\
\hline
\end{tabular}

${ }^{\mathrm{a}}$ Amino acid changed in index LHON family 
white-handed gibbon (Hylobates lar) showed the same amino acid sequence at the beginning of the COX2 gene as did the proband. However, given the significant evolutionary distance between the gibbon and modern humans (Hall et al. 1998; Gagneux et al. 1999), it is highly unlikely that the 7598A mutation would have the same significance for this hominoid ape species.

A mutation-specific restriction enzyme assay for the 7598A variant revealed that it was heteroplasmic in the proband and his mother, with mutational loads of 80 and 25\% mutant molecules, respectively (lanes IV-7 and

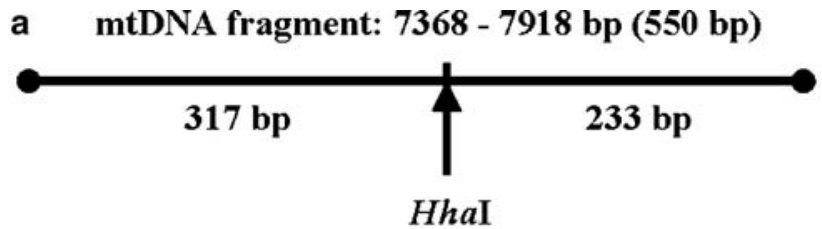

b

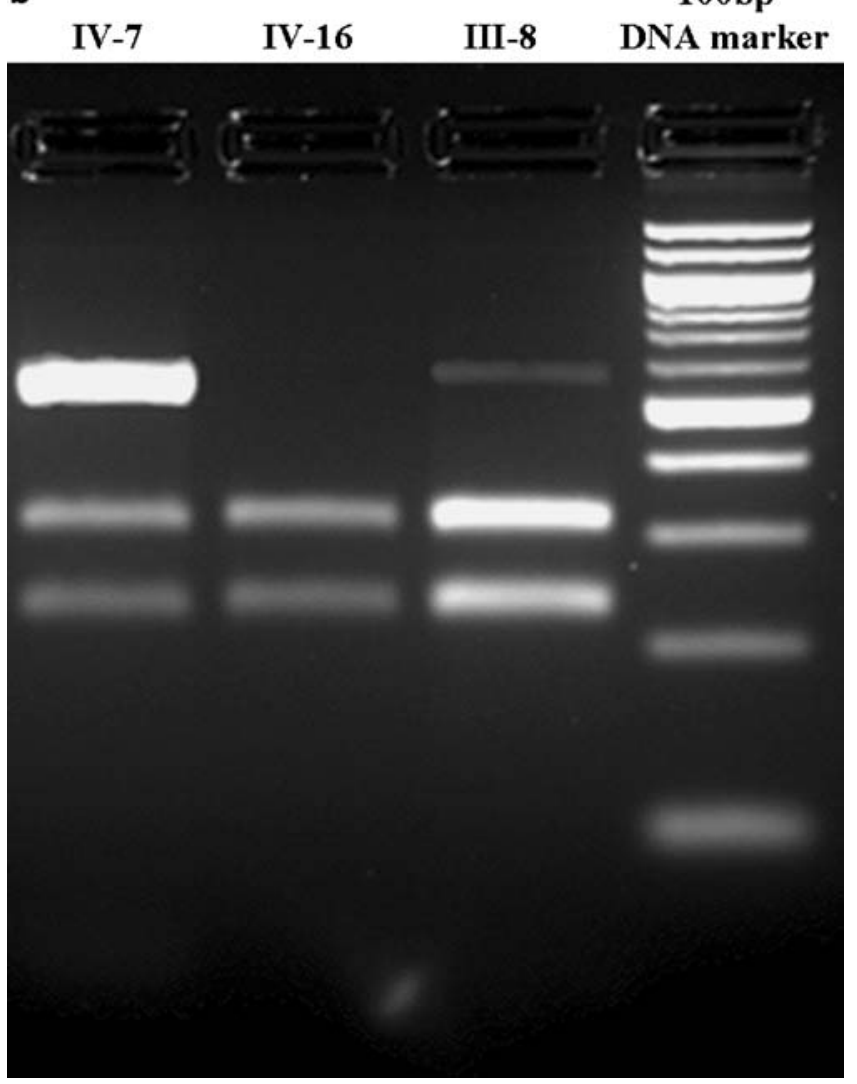

Fig. 3 a A schematic overview of the amplified fragment of the mtDNA indicates the position of the HhaI restriction site. The wild type mtDNA lacks the $H h a \mathrm{I}$ site and leads to a single fragment of $550 \mathrm{bp}$. The $7598 \mathrm{~A}$ mutation results in HhaI site gain and two fragments of 317 and $223 \mathrm{bp}$. b A short fragment including the cytochrome $c$ oxidase (COX2) gene (positions 7,368-7,918) was amplified in available family members, as designated on the gel image in concordance with pedigree symbols (Fig. 1). The fragments were resolved through agarose gel electrophoresis after $\mathrm{HhaI}$ digestion. The proband and his mother (lanes IV-7 and III-8, respectively) contain a mixture of mutant and wild type molecules; however, the proband's second cousin (lane IV-16) showed only wild type mtDNA molecules in his hair
III-8; Fig. 3). However, this mutation was totally absent from mtDNA isolated from the hair of the proband's cousin (lane IV-16, Fig. 3), who instead showed 100\% presence of the wild type allele. These data pointed to the de novo occurrence of the 7598A mutation in the proband's maternal lineage, most likely in the germ line of individual II-9. We also screened nearly 200 haplogroup-matched (haplogroup $\mathrm{H}$ ) normal and LHONpositive Caucasian controls from our data set for this mutation, but failed to detect it (data not shown).

In addition, we surveyed more than 1,000 published full mtDNA sequences of Caucasian origin available in GenBank (Torroni et al. 1996; Howell et al. 2003; Moilanen et al. 2003; Achilli et al. 2004; Coble et al. 2004), those available in the mtSNP Database, published reports of whole genome sequences (Ingman et al. 2000; Kivisild et al. 2002; Tanaka et al. 2004), and a list of unpublished polymorphisms (MITOMAP 2005; http:// www.mitomap.org) for the 7598A/COX2 mutation. We found two mtDNAs of Caucasian origin that also carried this mutation (Fig. 2). The mtDNA sequence LHON 4 (S015 in supplemental material of Howell et al. 2003) belonged to a 11778A-positive LHON patient whose mtDNA also contained a mutation set specific for haplogroup $\mathrm{H}$. This mtDNA also had the transversion at $14470 \mathrm{~A}$, which classifies it as belonging to sub-branch H10 (Achilli et al. 2004).

The sequence C5 (\#153) from Finnila et al. (2001) also carried the 7598A mutation at homoplasmic levels. It was obtained from an anonymous Red Cross donor participating in an occipital stroke survey of the Finnish population. This haplotype belongs to West Eurasian haplogroup $\mathrm{K}$, and differs from that of the proband by more than 20 SNPs (Fig. 2). Since neither of the published sequences was closely related to the proband's mtDNA or to each other, the 7598A mutation appeared to represent independent mutational events in different branches of the Caucasian mtDNA phylogeny (Fig. 2).

The 7598A mutation also appeared to be a monophyletic marker for East Eurasian haplogroup E. This maternal lineage occurs at low frequencies in some East and Southeast Asian populations (Stoneking et al. 1990; Ballinger et al. 1992; Torroni et al. 1994). However, it was not clear from these reports whether this particular mutation was truly present in all of these Asian haplotypes. They were reported as having the HhaI 7598 site loss, an RFLP marker that has been detected in different sub-branches of haplogroups $\mathrm{M}, \mathrm{N}$ and $\mathrm{R}$, and could potentially be caused by one of four different mutations in the HhaI recognition sequence (Ballinger et al. 1992; Torroni et al. 1994; Herrnstadt et al. 2002; Kivisild et al. 2002; Tanaka et al. 2004). Unfortunately, no whole genome sequence data were available for the earlier mtDNA studies (Ballinger et al. 1992; Torroni et al. 1994), making it uncertain if the reported RFLPs reflect the occurrence of the 7598A mutation.

Thus, for the sake of clarity, we restricted the assignment of haplogroup $\mathrm{E}$ to only those two individuals for whom full mtDNA sequences are available and 
in which the 7598A mutation is present (Herrnstadt et al. 2002; Ingman and Gyllensten 2003) (Fig. 2; haplotypes $\mathrm{C} 6$ and $\mathrm{C} 7$ ). One exception to this categorisation is a Japanese individual sequence in which the 7598A mutation appears, although it is clearly differentiated from haplogroup E mtDNAs by at least 20 mutations due to belonging to mtDNA haplogroup $\mathrm{C} 1$ (Tanaka et al. 2004).

It should further be added that the majority of forensic and population genetic studies often use genetic data from mitochondriopathy patients without any reference to the clinical or genealogical status of the examined individuals (e.g. Palanichamy et al. 2004; Achilli et al. 2004). As a result, such studies may erroneously bias estimates of frequency of deleterious mutations in population controls.

\section{Discussion}

We describe here evidence for the de novo occurrence of a heteroplasmic 7598A mutation in the COX2 gene of mtDNA in a Caucasian family affected with Leber's optic neuropathy. Although observed in this LHON maternal lineage, the presence of the 7598A mutation in control populations could potentially cast doubt on its pathogenic significance. However, its role as a deleterious variant in the proband's family can be supported by several lines of evidence.

First, the studied family exhibited the typical clinical presentation of the optic neuropathy, with a clear pattern of maternal phenotypic transmission. The clinical spectrum also included extra-ocular neurological changes that are normally seen in LHON patients when broad clinical investigatory tools are applied (Zhadanov 2000, 2001; Brown et al. 2001; Carelli et al. 2001). Although seizures and migraines are uncommon symptoms in LHON cases, these have been described earlier in the presence of primary mutations (e.g. Brown et al. 2001; Carelli et al. 2001), and could be also explained here by the nature of the genetic defect in the COX gene. The maternal inheritance of the clinical symptoms, along with the typical appearance of identical phenotypes in the first line of maternal cousins deriving from separate marriages of individual II-9 (Fig. 1), further suggest that the mitochondrial genome is the likely location of the defective gene.

Second, the 7598A mutation is heteroplasmic and present at high levels (mutant load $\sim 80 \%$ ) in the proband's blood cells. The confirmation of the de novo occurrence of this mutation in his maternal lineage clearly indicates its recent emergence in the affected family. This is an important observation because most phylogenetically neutral mutations rapidly segregate and become fixed during evolution of the mitochondrial genome, thereby creating the deep internal structure of the human mtDNA tree (Cann et al. 1987; Ingman et al. 2000; Herrnstadt et al. 2002). Unlike these variants, pathogenic defects are under certain negative selective pressure, and occur repeatedly during human evolution (Brown et al. 1995; Nachman et al. 1996; Torroni et al. 1997). As a result, they should be randomly distributed in different genetic backgrounds. Thus, the heteroplasmic appearance of the 7598A mutation may indicate its recent emergence in the proband's family, and likely points to its recurrence and elimination from human mtDNA genomes through negative selection throughout evolution.

Third, the load of mutated mtDNA molecules in the maternal pedigree corresponds very well with the phenotypic expression of the disease, assuming the existence of a specific genetic threshold for disease expression. In addition, those branches of the proband's family that had wild type alleles appeared not to express the deleterious phenotype, although no samples were available for members of the II12-V5 branch (Fig. 1) to test this assumption. However, while an important feature of this case, heteroplasmy may not be a necessary condition for defining LHON mutations (Smith et al. 1993), as only $12 \%$ of individuals carrying common LHON mutations demonstrate a mixture of mtDNA genomes (Man et al. 2003). In addition, we cannot exclude the existence of other low level heteroplasmic mutations in the studied mtDNAs that could segregate to significant levels in target tissues, as has been recently observed in Alzheimer's and Parkinson's patients (Coskun et al. 2004; Parker and Parks 2005).

Fourth, the 7598A mutation converts the evolutionarily poorly conserved hydrophobic aliphatic alanine into the hydrophilic polar threonine, in close vicinity to the evolutionarily stable domain of $\mathrm{COX} 2$, thereby enhancing the hydrophilicity of the adjacent polar glutamine. This amino acid change might be predicted to interfere with the anchoring of the $\mathrm{N}$-terminal transmembrane helix-hairpin of COX2 within the mitochondrial membrane (Tsukihara et al. 1996), which, in turn, would compromise the assembly or stability of the COX holoenzyme. In this regard, it is worth noting that many LHON mutations, including the primary $14484 \mathrm{C}$ transition, create substitutions at poorly conserved residues, which, being adjacent to conserved domains, can potentially lead to drastic defects and phenotypic expression in either mammals (LHON) or yeast models (Machingo et al. 2001; Kao et al. 2005).

Fifth, there is general agreement that deleterious mutations should not occur in healthy controls, nor should they be found at the population level as polymorphisms. However, an extensive survey of published mtDNA sequences revealed that the 7598A mutation has appeared independently at least three times in West Eurasian mtDNA backgrounds, and twice in different haplogroup $\mathrm{H}$ sub-branches, including the proband's mtDNA (Fig. 2). In both of these latter cases, when occurring in a haplogroup $\mathrm{H}$ background, the 7598A mutation was associated with a LHON phenotype (Fig. 2). Thus, it is possible that the 7598A mutation could be a contributing or modifying factor in LHON expression in the published family pedigrees. 
In a third case, the 7598A mutation apparently did not cause disease expression in its anonymous carrier, whose mtDNA belonged to haplogroup K (Finnila et al. 2001). However, the lack of phenotypic data makes it impossible to know whether this person developed a disease phenotype subsequent to blood donation (J.S. Moilanen, personal communication). On the other hand, none of the haplogroup $\mathrm{K}$ mtDNA sequences available through extensive population and forensic surveys, including our own 47 samples, have been shown to possess the 7598A mutation (Herrnstadt et al. 2002; Coble et al. 2004; unpublished data). Therefore, it is unlikely that the $7598 \mathrm{~A}$ mutation represents a phylogenetically ancient polymorphism in this haplogroup. Furthermore, haplogroup $\mathrm{K}$ has been associated with increased longevity, a protective effect against Alzheimer's and Parkinson's disease, and lowered LHON expression (Carrieri et al. 2001; van der Walt et al. 2003). For these reasons, this haplogroup could possibly tolerate the presence of deleterious mutations in its branches.

Similarly, a mtDNA from a female Japanese centenarian was also found to carry the 7598A mutation (TC52; Tanaka et al. 2004). Her mtDNA belonged to haplogroup $\mathrm{C}$, a maternal lineage in which mutations known to cause LHON or similar mitochondriopathies have not yet been detected. This observation, along with the woman's longevity, would seem to indicate that this mutation did not affect the function of the COX2 gene product. However, the exact interactions between the 7598A mutation and others present in the coding region of her mtDNA (e.g. 3826, 9099, 9730, 13590, 15973) are not known. This is an intriguing point because three of these mutations (3826, 9750 and 15773) cause non-synonymous changes in the ND1, COX3 and CytB genes, respectively. Therefore, it is possible that the deleterious effect of the COX2 mutation was ameliorated or buffered by the other mutations present in this Japanese mtDNA.

In light of these genetic data, one could ask whether the presence of a mutation in control population mtDNAs refutes its putative pathogenic role. Our findings suggest that, for LHON cases, it does not. Even severe LHON mutations often arise as singleton cases, and can be normally seen in healthy carriers in LHON families (Brown et al. 1995, 2001; Howell et al. 2003). Therefore, the rare occurrence or absence of known deleterious mtDNA mutations in normal controls may indicate the limited nature of genetic surveys of human populations, which would have undersampled individuals bearing these mutations, as well as reflecting the low penetrance of LHON in different human groups.

In fact, studies of large population cohorts have occasionally reported the presence of common LHON mutations in these groups (e.g. Herrnstadt et al. 2002; Howell et al. 2003; Palanichamy et al. 2004; Tanaka et al. 2004; Achilli et al. 2004). Moreover, genealogical analysis of LHON families with common 14484C mutation revealed them to have descended from a small number of founder haplotypes within haplogroup $\mathrm{J}$, thereby unambiguously demonstrating the fixation of deleterious mtDNA mutations in a phylogenetic lineage over its short evolution history (Howell et al. 2003; Laberge et al. 2005). The prolonged persistence of LHON mutations in a particular mtDNA background, such as haplogroup J, makes deleterious defects behave similarly to neutral variants, and blurs the distinction between pathogenic LHON mutations and mtDNA polymorphisms.

The 7598A COX2 mutation seems to demonstrate dual genetic characteristics of deleterious and neutral variants in behaving differently in separate branches of the human phylogenetic tree. This observation may be explained by the putative interaction of specific mtDNA variants with particular genetic environments. However, the nature of these interactions is not yet entirely clear. The 7598A mutation could possibly be another mildly deleterious mtDNA variant having adaptive significance in human evolution and accumulating during the ancient expansion of modern humans out of Africa as they relocated to new climatic zones (Mishmar et al. 2003). These initially 'advantageous' mtDNA variants may later have become maladaptive and detrimental when occurring as homoplasies in different mtDNA backgrounds.

Should this hypothesis be correct, it has at least two main implications. The first is that the relative neutrality of a given mutation should be determined with respect to the specific haplogroup background in which it occurs. In addition, for a mitochondrial disorder such as LHON to develop, a causative mutation must interact with certain 'neutral' variants within a particular genetic background. We believe that our finding of a putatively polymorphic $7598 \mathrm{~A}$ in association with a pathological phenotype in different genetic backgrounds supports this model, but are further investigating this relationship.

Acknowledgements We are grateful to our patients for their participation in this study. The authors also thank the administration of the Novosibirsk Branch of the Sv. Fyodorov State Institution, IRTC Eye Microsurgery, Novosibirsk, Russia, and Dr. Ludmila Osipova for providing control population samples. This work was supported in part by a Fight for Sight Grant-in-Aid and Faculty Research Funds from the University of Pennsylvania to T.G.S., and by research funds from the Sv. Fyodorov State Institution, IRTC Eye Microsurgery, Novosibirsk, Russia.

\section{References}

Achilli A, Rengo C, Magri C, Battaglia V, Olivieri A, Scozzari R, Cruciani F, Zeviani M, Briem E, Carelli V, Moral P, Dugoujon JM, Roostalu U, Loogväli EL, Kivisild T, Bandelt HJ, Richards M, Villems R, Santachiara-Benerecetti AS, Semino O, Torroni A (2004) The molecular dissection of mtDNA haplogroup $\mathrm{H}$ confirms that the Franco-Cantabrian glacial refuge was a major source for the European gene pool. Am J Hum Genet 75:910-919

Altschul SF, Madden TL, Schaffer AA, Zhang J, Zhang Z, Miller W, Lipman DJ (1997) Gapped BLAST and PSI-BLAST: a new generation of protein database search programs. Nucleic Acids Res 25:3389-3402 
Anderson S, Bankier AT, Barrell BG, de Bruijn MH, Coulson AR, Drouin J, Eperon IC, Nierlich DP, Roe BA, Sanger F, Schreier PH, Smith AJ, Staden R, Young IG (1981) Sequence and organization of the human mitochondrial genome. Nature 290:457-465

Andrews RM, Kubacka I, Chinnery PF, Lightowlers RN, Turnbull DM, Howell N (1999) Reanalysis and revision of the Cambridge reference sequence for human mitochondrial DNA. Nat Genet 23:147

Babcock GT, Wikstrom M (1992) Oxygen activation and the conservation of energy in cell respiration. Nature 356:301-309

Ballinger SW, Schurr TG, Torroni A, Gan YY, Hodge JA, Hassan K, Chen KH, Wallace DC (1992) Southeast Asian mitochondrial DNA analysis reveals genetic continuity of ancient mongoloid migrations. Genetics 130:139-152

Bandelt HJ, Forster P, Sykes BC, Richards MB (1995) Mitochondrial portraits of human populations using median networks. Genetics 141:743-753

Brown MD, Torroni A, Reckord CL, Wallace DC (1995) Phylogenetic analysis of Leber's hereditary optic neuropathy mitochondrial DNA's indicates multiple independent occurrences of the common mutations. Hum Mut 6:311-325

Brown MD, Zhadanov SI, Allen J, Hosseini S, Newman NJ, Atamonov VV, Mikhailovskaya IE, Sukernik RI, Wallace DC (2001) Novel mtDNA mutations and oxidative phosphorylation dysfunction in Russian LHON families. Hum Genet 109:33-39

Cann RL, Stoneking M, Wilson AC (1987) Mitochondrial DNA and human evolution. Nature 325:31-36

Carelli V, Valentino ML, Liguori R, Meletti S, Vetrugno R, Provini F, Mancardi GL, Bandini F, Baruzzi A, Montagna P (2001) Leber's hereditary optic neuropathy (LHON/11778) with myoclonus: report of two cases. J Neurol Neurosurg Psychiatry 71:813-816

Carrieri G, Bonafe M, De Luca M, Rose G, Varcasia O, Bruni A, Maletta R, Nacmias B, Sorbi S, Corsonello F, Feraco E, Andreev KF, Yashin AI, Franceschi C, De Benedictis G (2001) Mitochondrial DNA haplogroups and APOE4 allele are nonindependent variables in sporadic Alzheimer's disease. Hum Genet 108:194-198

Coble MD, Just RS, O'Callaghan JE, Letmanyi IH, Peterson CT, Irwin JA, Parsons T (2004) Single nucleotide polymorphisms over the entire mtDNA genome that increase the power of forensic testing in Caucasians. Int $\mathbf{J}$ Legal Med 118:137-146

Coskun PE, Beal MF, Wallace DC (2004) Alzheimer's brains harbor somatic mtDNA control-region mutations that suppress mitochondrial transcription and replication. Proc Natl Acad Sci USA 101:10726-10731

Darin N, Kadhom N, Briere JJ, Chretien D, Bebear CM, Rotig A, Munnich A, Rustin P (2003) Genotypes and clinical phenotypes in children with cytochrome- $c$ oxidase deficiency. Neuropediatrics 34:311-317

Finnila S, Hassinen IE, Majamaa K (2001) Phylogenetic analysis of mitochondrial DNA in patients with an occipital stroke. Evaluation of mutations by using sequence data on the entire coding region. Mutat Res 458:31-39

Gagneux P, Wills C, Gerloff U, Tautz D, Morin P, Boesch C, Fruth B, Hohmann G, Ryder O, Woodruff D (1999) Mitochondrial sequences show diverse evolutionary histories of African hominoids. Proc Natl Acad Sci USA 96:5077-5082

Hall ML, Jones DS, Wood BA (1998) Evolution of the gibbon subgenera inferred from cytochrome $b$ DNA sequence data. Mol Phylogenet Evol 10:281-286

Herrnstadt C, Elson JL, Fahy E, Preston G, Turnbull DM, Anderson C, Ghosh SS, Olefsky JM, Beal MF, Davis RE, Howell N (2002) Reduced-median-network analysis of complete mitochondrial DNA coding-region sequences for the major African, Asian, and European haplogroups. Am J Hum Genet 70:1152-1171

Howell N, Oostra RJ, Bolhuis PA, Spruijt L, Clarke LA, Mackey DA, Preston G, Herrnstadt C (2003) Sequence analysis of the mitochondrial genomes from Dutch pedigrees with Leber hereditary optic neuropathy. Am J Hum Genet 72:1460-1469
Ingman M, Gyllensten U (2003) Mitochondrial genome variation and evolutionary history of Australian and New Guinean aborigines. Genome Res 13:1600-1606

Ingman M, Kaessmann H, Paabo S, Gyllensten U (2000) Mitochondrial genome variation and the origin of modern humans. Nature 408:708-713

Johns DR, Neufeld MJ (1993) Cytochrome $c$ oxidase mutations in Leber hereditary optic neuropathy. Biochem Biophys Res Commun 196:810-815

Johns DR, Neufeld MJ, Hedges TR III (1994) Mitochondrial DNA mutations in Cuban optic and peripheral neuropathy. J Neuroophthalmol 14:135-140

Kao MC, Di Bernardo S, Nakamaru-Ogiso E, Miyoshi H, Matsuno-Yagi A, Yagi T (2005) Characterization of the membrane domain subunit NuoJ (ND6) of the NADH-quinone oxidoreductase from Escherichia coli by chromosomal DNA manipulation. Biochemistry 44:3562-3571

Kivisild T, Tolk HV, Parik J, Wang Y, Papiha SS, Bandelt HJ, Villems R (2002) The emerging limbs and twigs of the East Asian mtDNA tree. Mol Biol Evol 19:1737-1751

Laberge AM, Jomphe M, Houde L, Vezina H, Tremblay M, Desjardins B, Labuda D, St-Hilaire M, Macmillan C, Shoubridge EA, Brais B (2005) A "Fille du Roy" introduced the T14484C Leber hereditary optic neuropathy mutation in French Canadians. Am J Hum Genet 77:313-317

Liu VW, Zhang C, Linnane AW, Nagley P (1997) Quantitative allele-specific PCR: demonstration of age associated accumulation in human tissues of the $\mathrm{A} / \mathrm{G}$ mutation at nucleotide 3243 in mitochondrial DNA. Hum Mutat 9:265-271

Machingo Q, Mazourek M, Cameron V (2001) Second-site, intragenic alterations in the gene encoding subunit II of cytochrome $c$ oxidase from yeast can suppress two different missense mutations. Curr Genet 39:297-304

Man PY, Griffiths PG, Brown DT, Howell N, Turnbull DM, Chinnery PF (2003) The epidemiology of Leber hereditary optic neuropathy in the North East of England. Am J Hum Genet 72:333-339

Mishmar D, Ruiz-Pesini E, Golik P, Macaulay V, Clark AG, Hosseini S, Brandon M, Easley K, Chen E, Brown MD, Sukernik RI, Olckers A, Wallace DC (2003) Natural selection shaped regional mtDNA variation in humans. Proc Natl Acad Sci USA 100:171-176

Moilanen JS, Finnila S, Majamaa K (2003) Lineage-specific selection in human mtDNA: lack of polymorphisms in a segment of MTND5 gene in haplogroup J. Mol Biol Evol 20:21322142

Nachman MW, Brown WM, Stoneking M, Aquadro CF (1996) Nonneutral mitochondrial DNA variation in humans and chimpanzees. Genetics 142:953-963

Palanichamy MG, Sun C, Agrawal S, Bandelt HJ, Kong QP, Khan F, Wang CY, Chaudhuri TK, Palla V, Zhang YP (2004) Phylogeny of mitochondrial DNA macrohaplogroup $\mathrm{N}$ in India, based on complete sequencing: implications for the peopling of South Asia. Am J Hum Genet 75:966-978

Parker WD Jr, Parks JK (2005) Mitochondrial ND5 mutations in idiopathic Parkinson's disease. Biochem Biophys Res Commun 326:667-669

Smith KH, Johns DR, Heher KL, Miller NR (1993) Heteroplasmy in Leber's hereditary optic neuropathy. Arch Ophthalmol 111:1486-1490

Stoneking M, Jorde LB, Bhatia K, Wilson AC (1990) Geographic variation in human mitochondrial DNA from Papua New Guinea. Genetics 124:717-733

Tanaka M, Cabrera VM, Gonzalez AM, Larruga JM, Takeyasu T, Fuku N, Guo LJ, Hirose R, Fujita Y, Kurata M, Shinoda K, Umetsu K, Yamada Y, Oshida Y, Sato Y, Hattori N, Mizuno Y, Arai Y, Hirose N, Ohta S, Ogawa O, Tanaka Y, Kawamori R, Shamoto-Nagai M, Maruyama W, Shimokata H, Suzuki R, Shimodaira H (2004) Mitochondrial genome variation in eastern Asia and the peopling of Japan. Genome Res 14:1832-1850

Torroni A, Miller JA, Moore LG, Zamudio S, Zhuang J, Droma T, Wallace DC (1994) Mitochondrial DNA analysis in Tibet: 
implications for the origin of the Tibetan population and its adaptation to high altitude. Am J Phys Anthropol 93:189-199

Torroni A, Huoponen K, Francalacci P, Petrozzi M, Morelli L, Scozzari R, Obinu D, Savontaus ML, Wallace DC (1996) Classification of European mtDNAs from an analysis of three European populations. Genetics 144:1835-1850

Torroni A, Petrozzi M, D'Urbano L, Sellitto D, Zeviani M, Carrara F, Carducci C, Leuzzi V, Carelli V, Barboni P, De Negri A, Scozzari R (1997) Haplotype and phylogenetic analyses suggest that one European-specific mtDNA background plays a role in the expression of Leber hereditary optic neuropathy by increasing the penetrance of the primary mutations 11778 and 14484. Am J Hum Genet 60:1107-1121

Tsukihara T, Aoyama H, Yamashita E, Tomizaki T, Yamaguchi $\mathrm{H}$, Shinzawa-Itoh K, Nakashima R, Yaono R, Yoshikawa S (1996) The whole structure of the 13-subunit oxidized cytochrome $c$ oxidase at $2.8 \mathrm{~A}$. Science $272: 1136-1144$

Valentino ML, Barboni P, Ghelli A, Bucchi L, Rengo C, Achilli A, Torroni A, Lugaresi A, Lodi R, Barbiroli B, Dotti M, Federico
A, Baruzzi A, Carelli V (2004) The ND1 gene of complex I is a mutational hot spot for Leber's hereditary optic neuropathy. Ann Neurol 56:631-641

Walt van der JM, Nicodemus KK, Martin ER, Scott WK, Nance MA, Watts RL, Hubble JP, Haines JL, Koller WC, Lyons K, Pahwa R, Stern MB, Colcher A, Hiner BC, Jankovic J, Ondo WG, Allen FH Jr, Goetz CG, Small GW, Mastaglia F, Stajich JM, McLaurin AC, Middleton LT, Scott BL, Schmechel DE, Pericak-Vance MA, Vance JM (2003) Mitochondrial polymorphisms significantly reduce the risk of Parkinson disease. Am J Hum Genet 72:804-811

Zhadanov SI (2000) Clinical and genetic aspects of Leber's hereditary optic neuropathy (in Russian). Consilium 5:62-66

Zhadanov SI (2001) Leber's hereditary optic neuropathy: new insights (in Russian). Novoe v Ophthalmologii 2:28-37

Zhadanov SI, Atamanov VV, Zhadanov NI, Oleinikov OV, Osipova LP, Schurr TG (2005) A novel mtDNA ND6 gene mutation associated with LHON in a Caucasian family. Biochem Biophys Res Commun 332:1115-1121 